$\xi_{p}$

\title{
Silver Metallization Characterization and Resistivity Performance Potential toward Chemical Sensor Application
}

\author{
Amnani A. Bakar ${ }^{1}$, Nor H. Hashim²*, Hairul A. Tajuddin ${ }^{3}$, Mohd Z. Sahdan ${ }^{4}$, Faiz A. Saparudin ${ }^{5}$ \\ ${ }^{1,2,5}$ Faculty of Engineering Technology, University Tun Hussein Onn Malaysia, 84600 Panchor, Johor, Malaysia \\ ${ }^{3}$ Faculty of Science, University of Malaya, 50603 Kuala Lumpur, Malaysia \\ ${ }^{4}$ Faculty of Electrical and Electronic Engineering, University Tun Hussein Onn Malaysia, 86400 Parit Raja, Batu Pahat, Johor, \\ Malaysia. \\ *Corresponding author E-mail: haslina@uthm.edu.my
}

\begin{abstract}
Performance of limiting resistive and capacitive signal delays increases gradually depends on the complexity degree of multilayer metallization. Electrodes sensor for electrical applications requires low surface roughness and low resistivity metal layers. Amongst conductive metal series, silver ( $\mathrm{Ag}$ ) has the lowest resistivity. On the other hand, compared to aluminum and copper, this metal also has higher oxidation resistance. This study aims to characterize Ag thin film on the glass substrate and the resistivity performance by using a physical deposition technique for chemical sensor application. A series of Ag thin film with different thickness were prepared from thermal vacuum evaporator at $3.45 \times 10-5 \mathrm{~Pa}$ with applied current at 28 Ampere in 21 minutes. Four-point resistivity probing instrument was used for resistivity testing of the thin films with different thickness. The prepared Ag thin film shows a low average roughness at 1.89 $\mathrm{nm}$. A smooth and homogeneous of Ag thin film is an advantage to provide a sensitive surface for element recognition in the de velopment of chemical sensor and an adsorbate can be justified whereas it schematically assembled with the arrangement onto smooth and perfectly flat thin film surface. Ag thin film has shown a crystallite size with respect to $50.84 \mathrm{~nm}$. The low rough surfaces have fewer nucleation sites, therefore fewer grains (crystallites) will appear. The optimum thickness was determined at $107 \mathrm{~nm}$ and the resistivity of Ag thin film was an average at $1.988 \times 10-8 \mathrm{Ohm}$.
\end{abstract}

Keywords: a Thermal evaporator; resistivity; roughness; silver; thin film.

\section{Introduction}

Silver metallization and its potential as a favorable candidate for implementation as a future interconnect material for integrated circuit technology. Basically, Ag has a malleable metallic element, ductile and lustrous white with the atomic weight of $107.87 \mathrm{u}$. The melting point of $\mathrm{Ag}$ is $961{ }^{\circ} \mathrm{C}$ which is much higher that $\mathrm{Al}$ $\left(600{ }^{\circ} \mathrm{C}\right)$ [1]. Ag has reflected optical properties and also has a reduction of their antimicrobial activity [2]. Ag has much application in various fields including, electronic, sensor, optical [3], surface-enhanced Raman scattering [4], medicine [5] and environmental [6].

Amongst conductive metal series, silver (Ag) has the lowest bulk electrical resistivity at $1.57 \mu \Omega \mathrm{cm}$ at room temperature compared to other interconnection pure metal such as copper $(\mathrm{Cu})$ at $1.7 \mu \Omega$

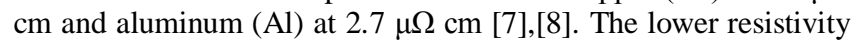
can reduce the RC delays and high power consumption. Ag also expected has higher electromigration $\left(0.95 \mathrm{eV}\right.$ at $\left.225-285^{\circ} \mathrm{C}\right)$ resistance compared to $\mathrm{Al}$ [9]. Ag has lower resistance than that of $\mathrm{Al}$, based on these reasons $\mathrm{Ag}$ is considered to be one of potential metallization schemes for future integrated circuits (IC's). The thermal stability of metallization and barrier layers become more critical for device reliability as feature sizes in multilevel metallization continue to shrink. Besides having low dielectric constant, the materials must have a good adhesion to silicon and to interconnect materials and thermal stability. But in anyhow, Ag thin film agglomeration has been observed on many substrates at high temperatures and considered as a drawback of silver metallization. The ability to deposit thin films of various materials is important for the fabrication of modern microelectronic devices and for enabling a variety of investigations of fundamental physical principles. The texture and the microstructure of thin metal films are closely related to the property of reliability and the structural of the thin film. Typically, the thin film layer structures, less than a micron in thickness, tailored to achieve the desired functional properties and with the better performance of interconnection in electrical properties.

Most techniques that fabricate $\mathrm{Ag}$ thin films are usually based on vacuum including evaporation, sputtering, and molecular beam epitaxy. Electrochemical deposition as an alternative for Ag deposition technique is important because of its affordability and flexibility. However, the mechanism of electrodeposition is complicated, because it is a non-equilibrium growth process and involves a large number of variables that influence the process including cation diffusion, $\mathrm{pH}$ and concentration of electrolyte and substrate type. The physical vapor deposition technique by using vacuum thermal evaporator instrument has a simplicity in experimental setup and cost-effective.

The objective of vacuum evaporation is to controllably of transfer atoms from a heated target. Basically, the thermal evaporation was operated with the target is heated directly or indirectly with the suitable pressure, power, and current until the point is reached where it efficiently sublimes or evaporates. 
In the present work, $\mathrm{Ag}$ thin films with various thicknesses from 10 to $230 \mathrm{~nm}$ have been prepared by physical vacuum deposition on unheated glass substrates. The structure, morphology and electrical properties of the films have been analyzed as a function of the layer thickness.

\section{Methodology}

The glass substrate was cleaned in a "piranha" solution, which is a mixture of $30 \% \mathrm{H}_{2} \mathrm{O}_{2}$ and concentrated $\mathrm{H}_{2} \mathrm{SO}_{4}(1: 3$, v/v) for $5 \mathrm{~min}$ at room temperature. Then, the glass substrate was rinsed copiously with deionized water and it will be ultra-sonicated thrice in deionized water to completely remove traces of sulfuric acid and ion. Afterward, the glass substrate was ultra-sonicated once in ethanol solution to remove undesirable particles, and dried under a stream of nitrogen gas as mentioned by [10], [11],

The experiment on the fabrication of $\mathrm{Ag}$ thin film was carried out to investigate the optimization of thickness in resistivity. This experiment will be carried out by using a thermal evaporator model of Ulvac Kiko VPC-061 (Figure 1) with the glass substrate $1 \mathrm{x}$ $1 \mathrm{~cm}$ (Figure 2).

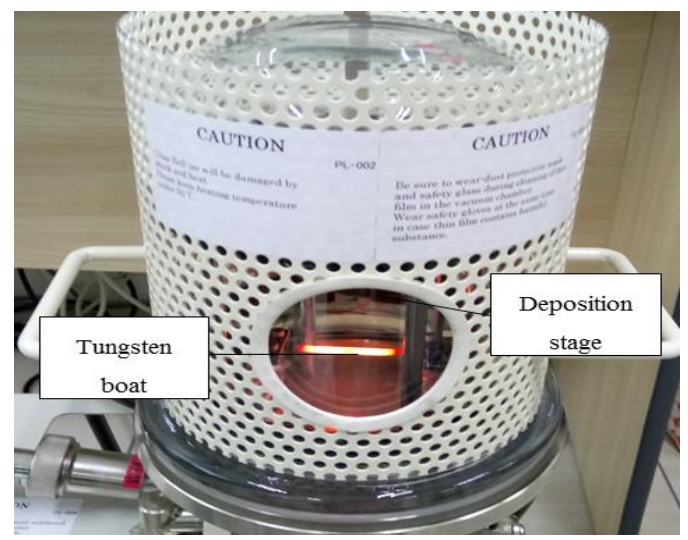

Fig.1: Thermal evaporator

Ag wire (99.999\% purity) was weighed using analytical balance and will be placed at the tungsten boat at thermal evaporator instrument. Then, the clean glass substrate was transferred immediately at thermal evaporator deposition stage. The current of deposition will be operated constantly at $3.45 \times 10-5 \mathrm{~Pa}$ with applied current at 28 Ampere in 21 minutes. The resistivity of different thickness at Ag thin film will be tested by using Four Point Resistivity Probing (model S-3024) instrument. The thickness of Ag thin film will be measured by using a surface profiler. The roughness of $\mathrm{Ag}$ was characterized by using Atomic-force microscopy (AFM) and the surface morphology of thin film was observed by using field emission scanning electron microscope (FESEM, JEOL7670) and the crystallinity of thin film was tested by using X-ray Diffraction (XRD).

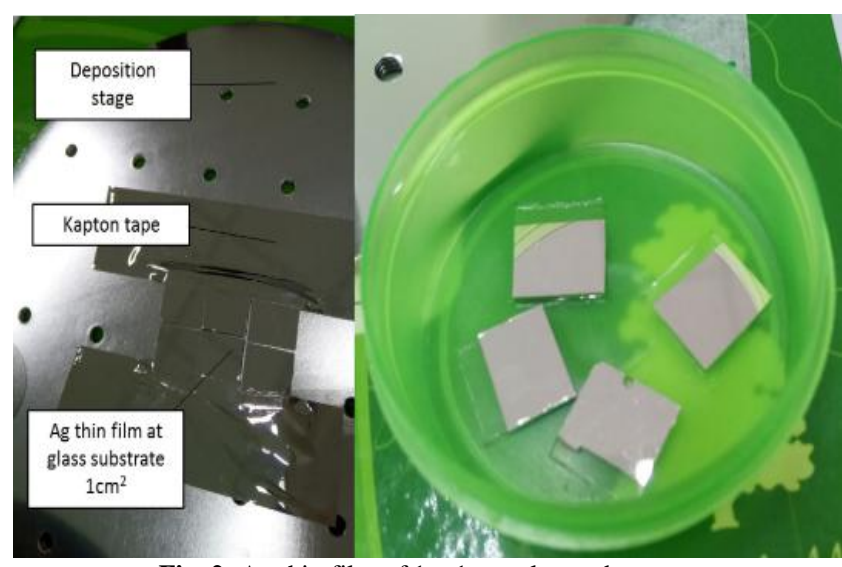

Fig. 2: Ag thin film of $1 \times 1 \mathrm{~cm}$ glass substrate.

\section{Result}

The morphology of Ag thin film on substrate glass was presented with the magnification at $50000 x$ by using FESEM image (Figure 3 ). In another word, this thin film was successfully deposited with $40.5 \mathrm{mg}$ at $5 \mathrm{~mm}$ length of $99.999 \%$ pure silver wire by using a physical deposition technique. The Ag thin film was prepared by the physical technique of thermal vacuum evaporator at a high and excellent pressure which is $3.45 \times 10^{-5} \mathrm{~Pa}$. The thickness of the $\mathrm{Ag}$ thin film was determined by surface profiler at $107 \mathrm{~nm}$.

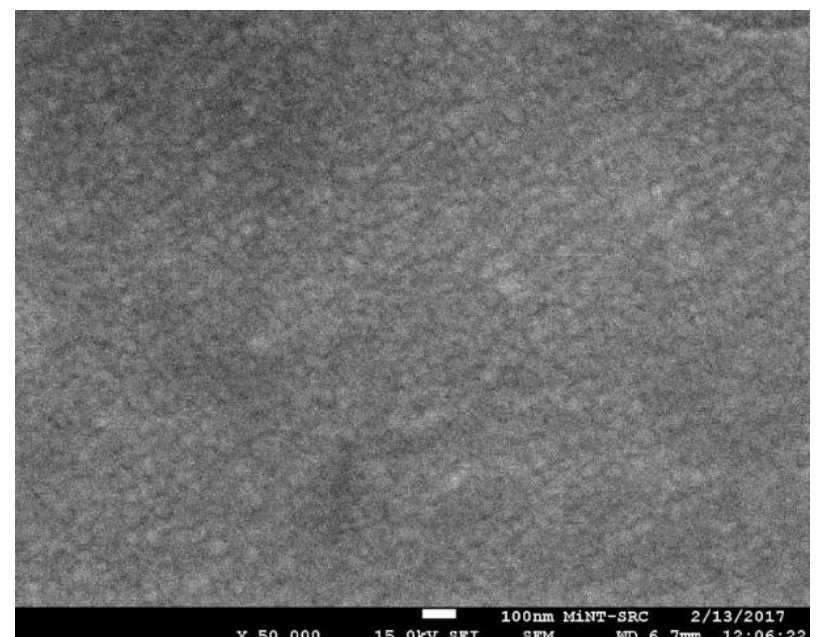

Fig. 3: Scanning electron microscopy micrographs of the image of the $\mathrm{Ag}$ thin film at $105 \mathrm{~nm}$

Additionally, Figure 4 shows the topography of $\mathrm{Ag}$ thin film by using AFM measurement image of Ag thin film on glass substrate. Indeed, the slow deposition of $\mathrm{Ag}$ thin film fabrication with a duration of 21 minutes was applied rather than rapid deposition with respect to current at 28 Ampere and the potential at $1.3 \mathrm{v}$. As a result, the slow deposition that is applied to the fabrication of $\mathrm{Ag}$ thin film shows a low roughness at $1.89 \mathrm{~nm}$ average roughness. Correspondingly, a smooth and homogeneous of $\mathrm{Ag}$ thin film is an advantage for Ag-SAM fabrication as the recognition element and the topography plays an important role towards a substrate which is a similar order or bigger than the size of the molecule adsorbate in the fabrication of integrated molecular assemblies as highlight by [12],[13].

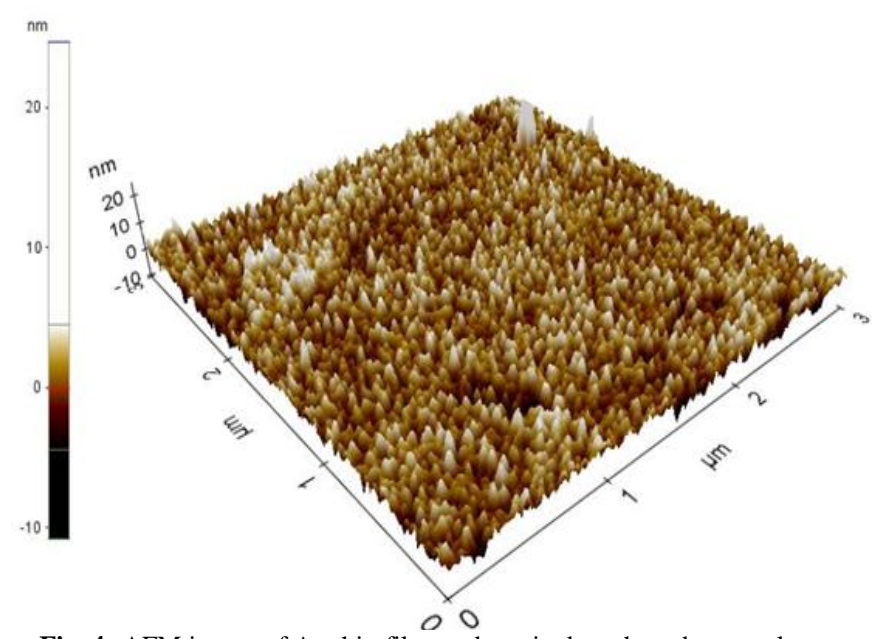

Fig. 4: AFM image of Ag thin film as deposited on the substrate glass

Meanwhile, the crystallinity from XRD instruments result of $\mathrm{Ag}$ thin film has shown a good crystallite size with respect to 50.84 $\mathrm{nm}$. Important to realize, that the crystallinity of the substrate sur- 
face becomes the essential role in determining the compactness, often quantitatively estimated by the pinholes distribution.

As revealed by the graph of mass Ag with thickness and resistivity in Figure 5, the thickness of Ag thin film was gradually increased with the increased of Ag mass. As a conductive element, Ag has good resistivity with an average of $1.988 \times 10^{-8} \mathrm{Ohm}$.m. The optimum of thickness Ag thin film was obtained with the interception of thickness and resistivity line which is $105 \mathrm{~nm}$ with $40 \mathrm{mg}$ of $\mathrm{Ag}$ mass.

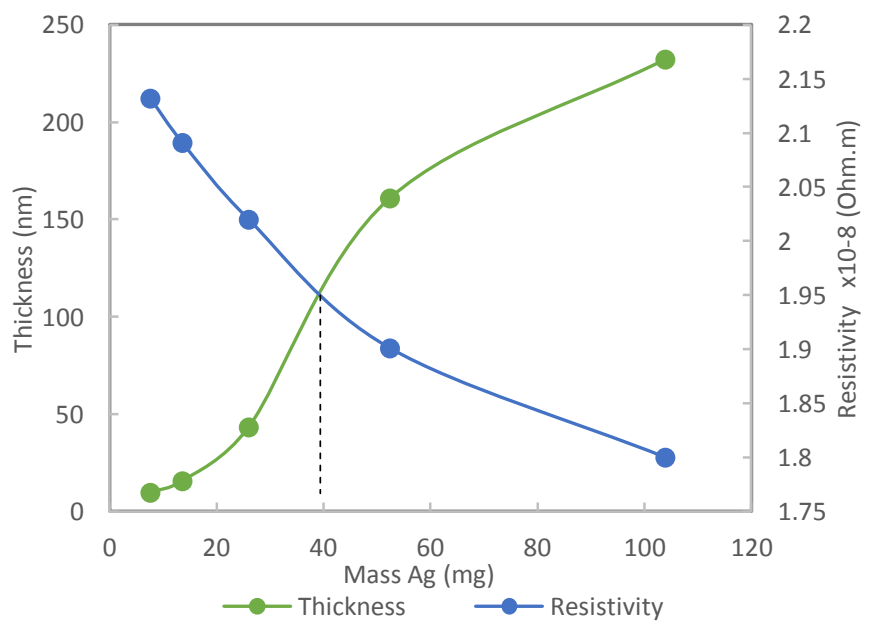

Fig. 5: Mass Ag with thickness and resistivity

The linear increase of resistivity is a result of electron scattering by lattice vibration. The resistivity of thinner films is lower than that of thicker films. The electrical resistivity changes dependence of the thickness of $\mathrm{Ag}$ on the glass substrate structure. The change in the morphology of $\mathrm{Ag}$ thin films also influence variations of the electrical resistivity which is an increase in resistivity due to increased surface scattering of conduction electrons [1].

\section{Conclusion}

From this study, it can be concluded that resistivity of silver thin films was studied using an in situ four-point probe technique. It was observed that film thickness plays an important role in the resistivity, which increased as the film thickness increased. Supersmooth metal films are particularly desirable for various electronic devices. The adhesion quality of deposited films onto substrates is directly dependent on the cleanliness of the substrate surface.

\section{Acknowledgement}

This work is funded by the Ministry of Higher Education of Malaysia under the Fundamental Research Grant Scheme (FRGS) Vot No.: 1565.

Special thanks to Microelectronics and Nanotechnology Shamsuddin Center (MiNT-SRC) for the facility.

\section{References}

[1] J. W. M. Daniel Adams, Terry L. Alford, Silver Metallization: Stability and Reliability. Verlag London: Springer-Verlag London, 2008.

[2] Y. Delgado-Beleño, C. E. Martinez-Nuñez, M. Cortez-Valadez, N. S. Flores-López, and M. Flores-Acosta, "Optical properties of silver, silver sulfide and silver selenide nanoparticles and antibacterial applications," Mater. Res. Bull., vol. 99, pp. 385-392, 2018

[3] H. Li, X. Yan, S. Qiao, G. Lu, and X. Su, "Yellow-Emissive Carbon Dot-Based Optical Sensing Platforms: Cell Imaging and Analytical Applications for Biocatalytic Reactions," ACS Appl. Mater. Interfaces, vol. 10, no. 9, pp. 7737-7744, 2018.

[4] L. Tao et al., "Silver nanoparticle-decorated graphene oxide for surface-enhanced Raman scattering detection and optical limiting applications," J. Mater. Sci., vol. 53, no. 1, pp. 573-580, 2018.

[5] T. S. Kim, J. R. Cha, and M. S. Gong, "Investigation of the antimicrobial and wound healing properties of silver nanoparticleloaded cotton prepared using silver carbamate," Text. Res. J., vol. 88, no. 7, pp. 766-776, 2018.

[6] X. Zhang, W. Wang, and D. Yu, "Synthesis of waterborne polyurethane-silver nanoparticle antibacterial coating for synthetic leather," J. Coatings Technol. Res., vol. 15, no. 2, pp. 415-423, 2018.

[7] H. C. Kim, T. L. Alford, and D. R. Allee, "Thickness dependence on the thermal stability of silver thin films," Appl. Phys. Lett., vol. 81, no. 22, pp. 4287-4289, 2002.

[8] K. Gardre and T. L. Alford, "Thermal stability and adhesion improvement of Ag deposited on Pa-n by oxygen plasma treatment," J. Vac. Sci. Technol. B, vol. 18, no. 6, pp. 2814-2819, 2000.

[9] Y. Zeng et al., "Texture and microstructural evolution of thin silver films in Ag/Ti bilayers," J. Appl. Phys., vol. 83, no. 2, pp. 779-785, 1998.

[10] G. March, T. Nguyen, and B. Piro, "Modified Electrodes Used for Electrochemical Detection of Metal Ions in Environmental Analysis," Biosensors, vol. 5, no. 2, pp. 241-275, 2015.

[11] J. Cancino, C. A. Razzino, V. Zucolotto, and S. A. S. MacHado, "The use of mixed self-assembled monolayers as a strategy to improve the efficiency of carbamate detection in environmental monitoring," Electrochim. Acta, vol. 87, pp. 717-723, 2013.

[12] J. C. Love, L. A. Estroff, J. K. Kriebel, R. G. Nuzzo, and G. M. Whitesides, Self-Assembled Monolayers of Thiolates on Metals as a Form of Nanotechnology. 2005.

[13] J. J. Gooding, F. Mearns, W. Yang, and J. Liu, "Self-Assembled Monolayers into the 21 st Century: Recent Advances and Applications," Electroanalysis, vol. 15, no. 2 2, pp. 81-96, 2003. 